\title{
Prognostic factors of Endometrial Cancer in a Cancer Hospital in Brazil
}

\author{
C. Anton, E.V.L. Mayerhoff, R.T. Kleine, J.P. Carvalho
}

ICESP - Instituto do Câncer do Estado de São Paulo

\section{Objectives}

To identify prognostic factors of endometrial cancer patients assisted in the Instituto do Câncer do Estado de São Paulo (ICESP), Brazil.

\section{Methods}

A retrospective analysis of 703 patients with endometrial cancer diagnosis referred to surgical treatment in ICESP between January 2009 and October 2016 was performed. Data has been exported from the REDCap database. Cox regression was used to identify parameters related to worse prognosis.

\section{Results}

The following parameters were included in the analysis: tumor histology, histologic grade, lymph-vascular space invasion (LVSI), tumor size (cutoff: 2 and $4 \mathrm{~cm}$ ) and myometrial infiltration. In the univariate analysis all studied parameters had $R R>2$. When multivariate analysis was performed the parameters with independent prognostic factors were histologic grade and LVSI (Table 1).

\section{Conclusion}

High grade tumors and the presence of lymph-vascular space invasion are worse prognostic factors in endometrial cancer patients.

Table 1: Endometrial cancer prognostic factors analysis

\begin{tabular}{|c|c|c|c|c|c|}
\hline \multicolumn{2}{|l|}{ Parameters } & Univariated (RR) & $\mathrm{p}$ & Multivariated (RR) & $p$ \\
\hline \multicolumn{2}{|c|}{ Tumor histology } & 3.1 & $p<0.0001$ & & \\
\hline \multicolumn{2}{|c|}{ Histologic grade } & 4.94 & $p<0.0001$ & 3.75 & $p<0.001$ \\
\hline \multicolumn{2}{|c|}{ LVSI } & 3.26 & $p<0.0001$ & 2.01 & $\mathrm{p}=0.001$ \\
\hline \multirow[t]{2}{*}{ Tumor size } & $2 \mathrm{~cm}$ & 2.46 & $\mathrm{p}=0.023$ & & \\
\hline & $4 \mathrm{~cm}$ & 2.25 & $p<0.001$ & & \\
\hline \multicolumn{2}{|c|}{ Myometrial invasion } & 2.78 & $p<0.001$ & & \\
\hline
\end{tabular}

